\title{
Music Festivals Serving as a Catalyst for Collaborative HIV Prevention Education and Expanded HIV Testing in Rural Uganda
}

\author{
Van Leewen $\mathrm{J}^{1,2,4}$, Nabimanya $\mathrm{H}^{3}$, Ward $\mathrm{A}^{1}$, Grundy $\mathrm{R}^{1}$, Thrun $\mathrm{M}^{1}$ \\ ${ }^{I}$ Global Livingston Institute, Denver, CO, USA \\ ${ }^{2}$ Woodrow Wilson International Center for Scholars, Washington, DC, USA \\ ${ }^{3}$ Reach a Hand Uganda, Uganda \\ ${ }^{4}$ University of Colorado Denver, CO, USA
}

\begin{abstract}
Objective: To demonstrate the applicability of a nontraditional approach to reaching high risk populations for HIV testing and reproductive health services in rural Uganda. Methodology: From 2014 through 2017, the Global Livingston Institute and its local partners produced a series of music festivals in semirural Uganda in order to facilitate HIV testing and reproductive health services offered by NGOs specializing in HIV and sexual health. Our aim was to assess the effectiveness of a music festival to engage persons in sexual health and HIV screening services. Clinical service data was compiled and analyzed. Findings: Between 2014 and 2017, over 82,000 persons attended the annual festivals and were exposed to HIV prevention messaging. Over 15,000 persons have been tested for HIV. In 2017, 8,098 HIV tests were performed. In addition, 533 people donated blood, 36 long-acting means of contraception were placed, 404 women were screened for cervical cancer, 2 tubal ligations were performed, and 103 men were referred for circumcision. Conclusion: Music festivals created a novel opportunity to provide sexual health services including prevention education, reproductive healthcare, and HIV testing to persons at risk for HIV in rural Uganda.
\end{abstract}

Key Words: HIV testing; rural Uganda; music festivals; community development; reproductive health

\section{Introduction}

Despite declines in new HIV infections over the last decade, it is estimated that $7.4 \%$ of all Ugandans are living with HIV (Uganda Ministry of Health, 2015) and that 83,000 people were diagnosed with HIV in Uganda in 2015 (UNAIDS, 2016). Notwithstanding routine HIV testing becoming more common, in 2011, the most recent year for which HIV testing estimates are available, it was estimated that $44 \%$ of men and $25 \%$ of women in Uganda had still never been tested for $\mathrm{HIV}^{3}$. Among adolescents and young adults aged 15-24, the prevalence of HIV in males was $2.3 \%$ in 2014, while for females it was 3.7\% (Uganda Ministry of Health, 2015). Unfortunately, many young adults do not seek HIV screening. Only $53 \%$ of women and $32 \%$ of men aged 15-24 who had sex in the last 12 months had an HIV test in the past year (Uganda Bureau of Statistics, 2011). Although region-specific data is lacking, it is thought that even fewer persons in rural settings in Uganda have been tested for HIV with many avoiding testing due to HIV-related stigma (AVERT, 2015).

Our formative research in rural areas suggested that many people refused to seek local HIV testing or other sexual health services out of fear of being perceived as sexually promiscuous or already living with HIV by their neighbors. In addition, many rural villages are lacking in health infrastructure. In order to obtain an HIV test, many young persons must travel to local towns and cities to seek out known prevention providers, yet few are motivated to do so. Many young Ugandans suggested that testing be offered in coordination with an "event" that would make a trip to a central town more worthwhile. By offering screening in a central location away from home villages, a music festival could mitigate some of the concerns about confidentiality while allowing easy access to expanded HIV screening in young populations that may not have otherwise chosen to seek testing.

Music festivals have been utilized elsewhere in Africa to draw persons to a central location for HIV screening (Beat AIDS Project Zimbabwe, 2017).

Corresponding author: Van Leewen J, Global Livingston Institute, and Woodrow Wilson International Center for Scholars. University of Colorado, Denver, CO, USA. Email: jamie@globallivingston.org

(ब) This article is distributed under the terms of the Creative Commons Attribution License, which permits unrestricted use and redistribution provided that the original author and source are credited. 
They not only allow prevention education to be delivered in a de-stigmatizing manner, they allow quasi-anonymity by facilitating easy access to HIV screening outside of home villages. Given their appeal to a younger population, they serve as an ideal means to draw youth and young adults to a central location where health, or other services, could be offered. In addition, by having HIV and sexual health prevention messages delivered by popular music artists serving as champions, a music festival could normalize such prevention messaging for the young audience attending. Yet, to our knowledge, nothing has been published on the effectiveness of music festivals to deliver HIV prevention and screening services.

We hypothesized that a music festival in rural Uganda would serve as an effective catalyst to draw people, particularly young adults, to a central location where prevention education and HIV testing could be conveniently offered to those at risk for HIV.

\section{Methods}

Over several years, GLI developed relationships with several national and local organizations that synergized to develop and implement the music festivals. A U.S.-based lead agency organized the logistics of the festivals, coordinated planning and evaluation, and facilitated fundraising. A Ugandan national sexual health and youth advocacy organization oversaw outreach and marketing. In September 2014 and 2015, these two NGOs partnered to produce an annual one-day music festival in Kabale, Uganda, the I Know Festival. Kabale is located in Southern Uganda approximately 35 kilometers from the Rwandan border.

According to the Kabale District local government, the population of the district is projected to be 490,227 (228,546 and 261,681 male and female respectively) (Kabale District Local Government Statistical Abstract, 2012). Out of these, only 45,892 stay in the Municipality and the remaining $91 \%$ stay in the rural area. The District is densely populated with population density approximated in 2009 to be at 318 people per $\mathrm{km}^{2}$ (824 people per mile ${ }^{2}$. The land is heavily fragmented with an average household having six to seven plots of land on several hills. The people are predominantly from Bantu ethnic group and these are the Bakiga tribe, Batwa (Pygmies), the Banyarwanda and the Bahororo. The average household size in Kabale District is 4.8, which is high compared with other districts of Bushenyi, Kasese and Masindi in Western Uganda (Kabale District Local Government Statistical Abstract, 2012).

In September 2016, the festival expanded to include Lira, Uganda and testing was also offered on the day prior to the festival in each of the two locations. Everyone residing in the region of the festivals was welcomed to attend, but there was an intentional marketing emphasis on reaching youth and women with HIV testing and reproductive health services. The festivals engaged popular Ugandan and American music artists on a volunteer basis to not only perform in the festival, but also deliver prevention messages that focused on the importance of knowing one's HIV status and getting tested. In September 2017, the festival expanded to include Masaka, Uganda following the identical model as in Lira the year prior.

Prevention messaging was delivered via print, social media, radio and television marketing leading up to the festival, as well as verbally from the artists performing onstage during the festival. Local branches of sexual health-focused NGOs and clinician-intraining student groups volunteered their HIV screening and education services as well as onsite reproductive health services, such as PAP screening and IUD insertion. A blood bank performed HIV testing while recruiting blood donations.

Agencies reported HIV and other screening data to a central database. Our staff reviewed this data and sought to clarify any missing data. We present herein simple qualitative descriptions and frequency data based on agency reporting.

\section{Results}

Between 2014 and 2017, over 82,000 persons attended the annual festivals and were directly exposed to HIV prevention messaging in print leading up to the festival and verbally during the festivals. Over 1,000,000 people have seen social media impressions and over $9,000,000$ were exposed to prevention messages on television in Uganda. 
Table 1: Testing and Attendance at the iknow Concert Series, 2014-17

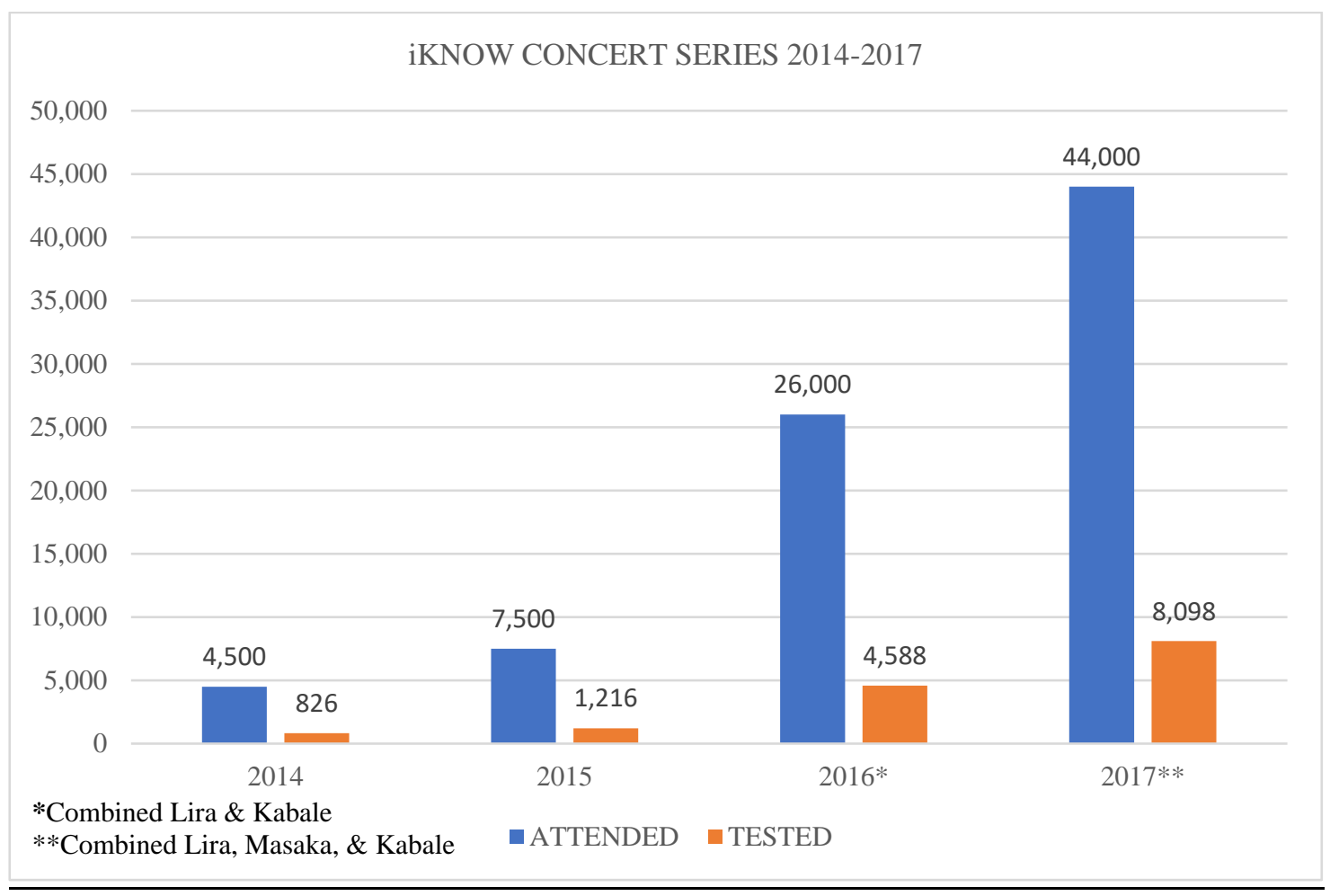

Over 15,000 persons have been tested for HIV since 2014. In 2017 alone, 44,000 people were estimated to have attended the festivals and 8,098 HIV tests were performed (representing $18.4 \%$ of all attendees); $56 \%$ of those tested were under the age of 25 and $34 \%$ tested were women. All those testing positive, $1.6 \%$ of persons screened, were offered linkage to care services. In addition, 36 long-acting means of reversible contraception - IUDs or implants - were placed during the festival, 404 women were screened for cervical cancer, 2 tubal ligations were performed, and 103 men were received circumcisions.

Following the festival in 2016, a sexual health collaborative was formed in Kabale, Uganda. Each of the agencies performing health services at the Kabale site of the festival has attended monthly meetings in collaboration with several additional health service providers. These meeting have been structured to offer both operational and educational support to local health agencies. Agencies have used the meetings to plan for future health screening opportunities, such as subsequent music festivals, but also including smaller health outreaches. Each meeting has also included a health presentation by one of the partner agencies in order to increase mutual proficiency on local health issues, specifically in the sexual health realm.

\section{Discussion}

Healthcare access is limited in rural Uganda. When overlaid with concerns about stigma and confidentiality, access to sexual health services is even more restricted. In order to stem the HIV epidemic in resource poor settings, novel means of sexual health service delivery are needed. By encouraging people in rural settings to gather in one location alongside service providers of sexual health services in the context of the iKnow Festivals, we were able to successfully screen over 15,000 people for HIV while directly educating tens of thousands in a de-stigmatized manner on the importance of HIV prevention and keeping oneself sexually healthy.

Intentionally, we did not attempt to provide health services directly. Rather, we partnered with many incountry organizations with clinical expertise in the delivery of sexual health and HIV prevention services. For several organizations, the services delivered during the music festivals represented a significant proportion of their planned annual service delivery goals. As such, these organizations are eager to continue to partner to provide services in the context of the festivals. Relationships with healthcare providers established in the context of the festivals have now 
developed into year-round efforts to better educate local clinicians on sexual health services and expand the provision of sexual health services throughout the year.

The music festivals were successful, but there remain areas for improvement. Women represented only a third of those testing for HIV. Efforts are needed to encourage more women to seek sexual health services in the context of the festival; plans for 2018 include an expansion of health services for women and intentional outreach to women prior to the concerts. The concert itself required funding to implement, but the direct and indirect economic impact for the region was significant. We are currently working to establish a plan to re-direct this investment back into future events and health services throughout the rest of the year.

Given ongoing challenges to the sustainable provision of sexual health services in many countries, music festivals may serve as a novel facilitator. Additional research is needed to better assess the realworld effectiveness and reach of music festivals to provide needed HIV prevention and education services. The music festivals appear to be serving two key roles for the local community. They are an economic development engine that is providing a forum for the community to come together and actively share music and culture in a setting that is intentionally designed to promote health and wellness. As a node for convening they also appear to be providing a platform for the local health providers to better collaborate and deliver services. The broader learnings from this study suggest that this model could be a mechanism not only to reach high risk populations in hard to reach rural communities but could also be a catalyst for collaboration and information sharing for local health providers.

\section{Conclusion}

By engaging local and international artists to share music and culture in communities where such opportunities are rare, the music festival appears to be a low cost way to assemble high risk persons in rural Uganda to provide HIV testing and reproductive health services. This model should be further examined for its capacity to scale to other communities. Opportunities for other public health interventions through music festivals are possible and warrant further research and investigation.

\section{Funding}

Primary funding for this project was provided by the US mission in Uganda and the AIDS Healthcare Foundation.

\section{Declaration of Conflicting Interests}

The author(s) declared no potential conflicts of interest with respect to the research, authorship, and/or publication of this article.

\section{Acknowledgment}

The authors wish to acknowledge the following organizations for contributing to this project: The Wilson Institute, Odyssey House Louisiana, Reproductive Health Uganda, AIDS Information Center, Marie Stopes, Kigezi Healthcare Foundation, the Kabale Institute for Health Sciences, Uganda Blood Bank, and the Lira School of Comprehensive Nursing.

\section{References}

AVERT - ABC of HIV Prevention. (2016, December 22). Retrieved May 3, 2017, from https://www.avert.org /professionals/hiv-around-world/sub-saharanafrica/uganda\#footnote27_b58ojx4

Beat AIDS Project Zimbabwe. (n.d.). Retrieved May 03, 2017, from http://whcbapz.org/

Kabale District Local Government Statistical Abstract (2012). Retrieved from http://www.ubos.org/ onlinefiles/uploads/ubos/2009_HLG_\%20Abstract_pri nted/CIS+UPLOADS/Higher\%20Local\%20Governme nt\%20Statistical\%20Abstracts_2012/Kabale.pdf

Prevention Gap Report - UNAIDS. (2016, July 11). Retrieved May 3, 2017, from http://www.unaids.org /sites/default/files/media_asset/2016-prevention-gapreport_en.pdf

Uganda Demographic and Health Survey 2011. (2012, August). Retrieved May 3, 2017, from https://www.usaid.gov/sites/default/files/documents/1 860/Uganda_Demographic_and_Health_Survey_2011 .pdf

Uganda HIV and AIDS Country Progress Report 2014 Uganda Ministry of Health. (2015, June 15). Retrieved May 3, 2017, from http://www.unaids.org/sites/default/ files/country/documents/UGA_narrative_report_2015. pdf 